\title{
Perioperative Management of Sectio Caesarea Surgery in Patient With Heart Valve Disorders \\ Purwoko ${ }^{*}$, Zidni Afrokhul Athir. ${ }^{{ }^{*}}$
}

Article Info :

Submitted :

17-03-2021

Accepted :

23-04-2021

Published :

26-04-2021

https://doi.org/10.20961/soja.v1i

1.49474

Authors' affiliations :

"Department of Anesthesiology and Intensive Therapy, Medical

Faculty, Universitas Sebelas

Maret, Surakarta, Indonesia

${ }^{\circledR}$ Correspondence:

zidniafrokhul@gmail.com

\begin{abstract}
Cardiovascular disease in pregnancy is common range from $1 \%$ to 3 and contributes to $10-15 \%$ of maternal mortality. Valvular heart disease accounts for about $25 \%$ of cases of cardiac complications in pregnancy and important cause of maternal mortality, some of which are mitral stenosis and mitral regurgitation. Cesarean delivery remains the preferred choice, as it reduces the hemodynamic changes that can occur in normal delivery and allows for better monitoring and hemodynamic management. Our paper provide in-depth information regarding the pathophysiology of heart valve disease in pregnant women and an appropriate perianesthesia approach to obtain a good prognosis. We report a case of a 26-year-old pregnant woman, with obstetric status G1P0A0, 36 weeks' gestation, body weight $61 \mathrm{~kg}$ accompanied by severe mitral regurgitation and moderate mitral stenosis. This patient was planned to undergo elective cesarean section. The patient's condition in the perioperative examination was: GCS E4V5M6, other vital signs within normal limits, $\mathrm{SpO} 2$ $98-99 \%$ in supine position. Other physical and laboratory examinations were also within normal limits. The goal of anesthesia during surgery in patients with heart valve disease undergoing cesarean section maintain pulmonary capillary pressure to prevent acute pulmonary edema. In this case, regional anesthesia of epidural anesthesia was chosen because it can reduce systemic vascular resistance and provide better postcesarean section pain. The patient's hemodynamics perianesthesia tended to be stable without any complications such as pulmonary edema.
\end{abstract}

Keywords: mitral stenosis; mitral regurgitation; obstetric anesthesia; sectio caesarea 


\section{INTRODUCTION}

Pregnant women have hemodynamic changes in the form of increased blood volume up to $40 \%$ compared to before pregnancy. This physiologic changes in blood volume is due to increased fetal and maternal metabolic requirements, particularly due to the influence of the uteroplacental circulation ${ }^{(1,2)}$. One of the most common pregnancy complications is cardiovascular disease, which accounts for $1 \%$ to $3 \%$ of pregnancy rates and contributes to $10 \%$ to $15 \%$ of maternal mortality Caesarean section delivery is still the main choice because it reduces the hemodynamic changes that can occur in normal delivery and allows for better monitoring and hemodynamic management ${ }^{(2,3)}$.

Valvular heart disease accounts for about $25 \%$ of cases of cardiac complications of pregnancy and the leading cause of maternal mortality. Examples of heart valve abnormalities are Mitral Stenosis (MS) and Mitral Regurgitation (MR) (2). Valve abnormalities of mitral stenosis and physiological changes in pregnancy predispose to acute pulmonary edema due increased pulmonary capillary pressure ${ }^{(4,5)}$. Acute pulmonary edema in pregnancy is a lifethreatening event and increases morbidity and mortality significantly ${ }^{(5,6)}$. Mitral regurgitation in pregnancy can cause volume overload in the left ventricle ${ }^{(2)}$. The management of anesthesia is based on the severity of the valve abnormalities and the hemodynamic changes associated with the surgical procedure ${ }^{(7)}$.

Until now, case reports of perioperative management of obstetric patients with valvular heart disease undergoing cesarean section limited. This is the background for the authors able to collect more detailed case data related to perianesthesia management of patients with severe mitral regurgitation and moderate mitral stenosis who will undergo cesarean section surgery.

\section{CASE ILLUSTRATION}

A pregnant woman, named Mrs. G, 26 years old, with obstetric status G1P0A0, 36 weeks of gestation with body weight $61 \mathrm{~kg}$ was planned for elective cesarean section. The patient was referred from the general hospital Assalam Gemolong with vitium cordis information. The patient feels that she is 9 months pregnant, does not complain about regular urination, blood mucus, or amniotic fluid. The patient still feels active fetal movements. The patient feels short of breath during strenuous activity, but the tightness gets better with rest and does not complain of chest pain. The patient feels tired easily, often wakes up at night due to shortness of breath. The patient is currently coughing, but is not accompanied by fever, 
runny nose, shortness of breath, chest pain, or blurred vision.

\section{Physical examination revealed} Glasgow Coma Scale E4V5M6, as well as other vital signs within normal limits. On saturation examination, the $\mathrm{SpO} 298 \%$ in all four extremities in the supine position. On cardiovascular examination, an irregular I-II heart sound was found, and a systolic roar was found at the apex grade 3/6. The laboratory showed Hemoglobin value of 12.5 g\%. Hematocrit 37\%, and Leukocytes 6,600 / $\mathrm{mm}^{3}$. Electrocardiography obtained sinus rhythm with a heart rate of 98 beats per minute accompanied by frequent Premature Ventricular Contraction (PVC). On echocardiography examination, found the dimensions of the Left Ventricle (LV) dilatation, IVS and $\mathrm{P}$ wave were abnormal, the mass did not increase; Low normal LV contractility Ejection Fraction (EF) 51\%; Wall Motion: Global Normokinetics; Dimensions of Left Atrium (LA) dilated, Right Atrium (RA) \& Right Ventricle (RV): Normal; RV contractility was good with TAPSE $2.8 \mathrm{~cm}$. In the valves of the heart, aorta: 3 cusps within normal limits; Mitral: there was thickening of the valve tip, severe Mitral Regurgitation (MR) with VCW 0.9 $\mathrm{cm}$, ERO $0.7 \mathrm{~cm}$, moderate Mitral Stenosis (MS) with MVA by plan $1.1 \mathrm{~cm}$, By PHT 1.4 $\mathrm{cm}$, By VTI $1.2 \mathrm{~cm} 2$ (score 2.2.2.2);
Tricuspid: moderate Tricuspid Regurgitation (TR) with VCW $0.4 \mathrm{~cm} 2$, ERO $0.2 \mathrm{~cm}$, TR Vmax 3.47 m / s; Pulmonary: Within Normal Limits, mPAP $43 \mathrm{mmHg}$, high probability of Pulmonary Hypertension (PH). Conclusion echocardiography showed severe MR, moderate MS, moderate TR, and low normal LV contractility EF 51\% high probability $\mathrm{PH}$. Abdominal ultrasound examination showed placenta insertion in the anterior body, enough amniotic fluid was visible, and no major congenital abnormalities were evident.

After obtaining medical approval from the family, the patient was transferred to the operating room and monitored according to ASA recommendations. The patient's blood pressure measured 135/86 $\mathrm{mmHg}$, pulse rate 109 beats per minute, and $\mathrm{SpO} 2$ $98 \%$.

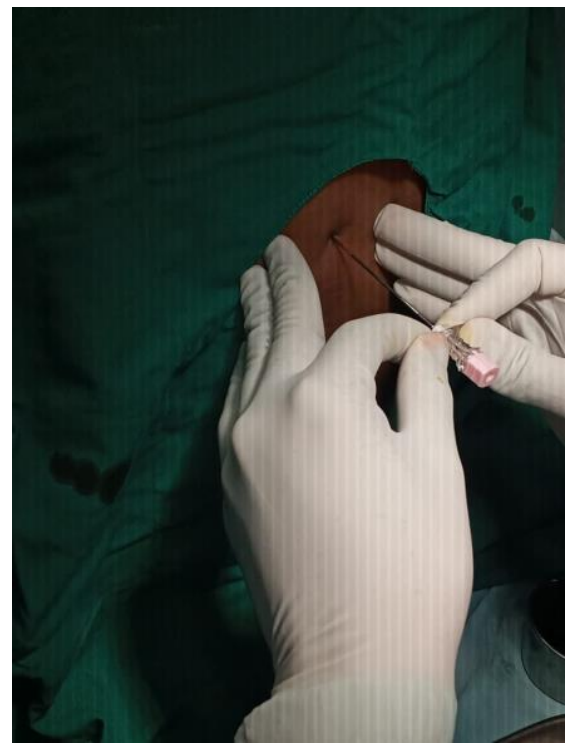

Figure 1. Epidural Anesthesia 
The patient was given 300cc crystalloid fluid. Epidural induction was performed using Levobupivacaine $0.375 \%$ and Fentanyl $50 \mathrm{mcg}$ with total volume $12 \mathrm{cc}$. During the operation, the patient received an intravenous injection of Tranexamic Acid 1 $\mathrm{g}$, Dexamethasone $5 \mathrm{mg}$ iv, Ondansetron 8 mg iv, and Oxytocin 10 IU drip. The patient was placed in an epidural puncture at L1-L2, tip at T11, T12. The patient is also fitted with a Foley catheter to monitor urine production.

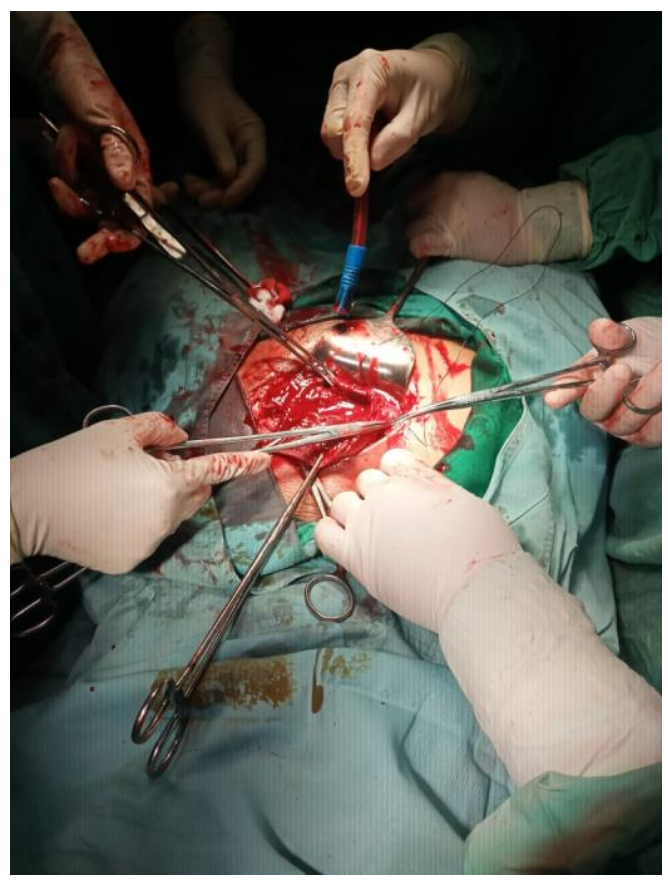

Figure 2. Sectio Caesarean

The patient's hemodynamic was stable during surgery which lasted 55 minutes. Hemodynamics during surgery were as follows: heart rate ranges from $80-110$ beats per minute, systolic blood pressure 110-140 $\mathrm{mmHg}$, diastolic blood pressure 70-90 $\mathrm{mmHg}, \mathrm{SpO} 299-100 \%$ with a nasal cannula
$3 \mathrm{lpm}$. During the operation, bleeding was obtained about $300 \mathrm{ml}$. The patient received postoperative epidural analgesia in the form of Levobupivacaine $0.125 \%$. The patient was then transferred to the ICU for intensive monitoring.

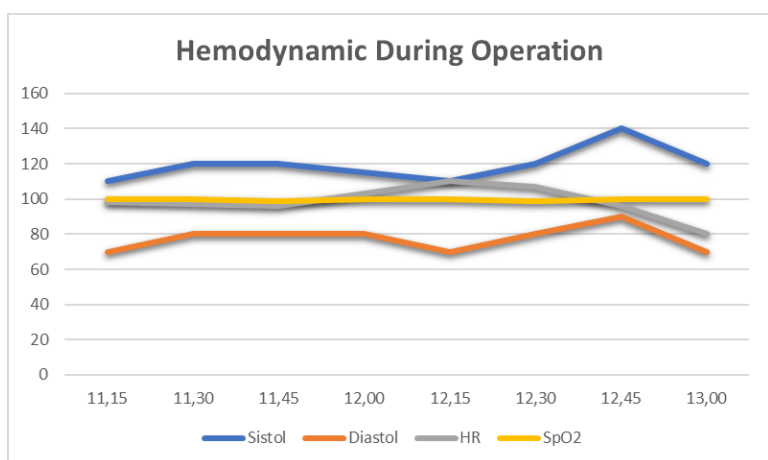

Figure 3. Hemodynamic Charts During Operation

\section{DISCUSSION}

The primary objective of anesthetic procedures in patients with severe $M R$ and moderate MS undergoing cesarean section is to maintain pulmonary capillary pressure to avoid the event of acute pulmonary edema ${ }^{(4)}$. Therefore, the use of general anesthesia should be avoided as it is associated with increased pulmonary pressure in response to laryngoscopy and intubation ${ }^{(8)}$. Regional anesthesia is an option in this case because it may reduce systemic vascular resistance and the workload of the left ventricle ${ }^{(8)}$. Epidural anesthesia has an advantage over spinal anesthesia because it provide better control of post-caesarean section pain ${ }^{(9)}$. 
Levobupivacaine has lower toxicity than Bupivacain, thereby reducing the risk of decreased contractile function, arrhythmias, cardiovascular collapse, and death ${ }^{(10,11)}$. Also, Levobupivacaine can provide sensory and motor blocks similar to bupivacaine, but with lower toxicity ${ }^{(11)}$. The characteristics of Levobupivacaine make this local anesthetic become the main choice in obstetric cases because pregnant women have a higher risk of toxicity due to physiological adaptations ${ }^{(11)}$.

Fentanyl is used as an adjuvant to Levobupivacaine to accelerate the onset, increase the duration of sensory and motor blocks, and provide fewer side effects, and hemodynamic stability as postoperative analgesica $^{(12)}$. Beside, fentanyl has a lower hypotensive effect compared to other opioids $^{(13) .}$

Decreased functional residual capacity and increased oxygen demand in patients were been treated by administering oxygenation using a $3 \mathrm{lpm}$ nasal cannula to prevent desaturation. This desaturation can lead to arrhythmias and respiratory failure $(14,15)$. Adequate rehydration is given to the patient to maintain optimal perfusion and oxygen delivery to the tissues ${ }^{(16)}$. Care should be taken to monitor fluid administration to prevent fluid overload ${ }^{(17)}$. In this case, we administered post-caesarean analgesia in the form of Levobupivacaine to reduce post- cesarean pain, which can cause impaired milk production in patients and reduced quality of neonatal care ${ }^{(18)}$.

\section{CONCLUSION}

Perioperative management of pregnant patients with complex valvular heart disease undergoing cesarean section requires an understanding of their pathophysiology and appropriate perianesthesia approach to achieve a good outcome.

\section{REFERENCE}

1. Talbot L. Physiology of pregnancy. Anaesth Intensive Care Med [Internet]. 2016; Available from: http://dx.doi.org/10.1016/j.mpaic.2016.04. 010

2. Anthony J, Osman A, Sani MU. Valvular heart disease in pregnancy. Cardiovasc $\mathbf{J}$ Afr. 2016;27(2):111-8.

3. Arendt KW, Lindley KJ. Obstetric Anesthesia Management of the Patient with Cardiac Disease. Int J Obstet Anesth [Internet]. 2018; Available from: https://doi.org/10.1016/j.ijoa.2018.09.011

4. Iqbal MA, Gupta M. Cardiogenic Pulmonary Edema. StatPearls. 2020.

5. Sciscione AC, Ivester $\mathrm{T}$, Largoza $\mathrm{M}$, Manley J, Shlossman P, Colmorgen GHC. Acute pulmonary oedema in pregnancy. Obstet Gynaecol Forum. 2017;27(2):4-9.

6. Righini M, Robert-EH, Le GG. Diagnosis of acute pulmonary embolism. 2017;1251- 
61.

7. Brown ML, Dinardo JA. Post-Operative Outcomes in Children With and Without Congenital Heart Disease Undergoing Noncardiac Surgery. 2016;67(7).

8. Sen S, Chatterjee S, Mazumder $\mathrm{P}$, Mukherji S. Epidural anesthesia: A safe option for cesarean section in parturient with severe pulmonary hypertension. J Nat Sci Biol Med. 2016;7(2):182-5.

9. Rehatta NM, Hanindito E, Tantri AR, Redjeki IS, Soenarto RF, Bisri DY, et al. Anestesiologi dan Terapi Intensif: Buku Teks KATI-PERDATIN. 1st ed. Jakarta: PT. Gramedia Pustaka Utama; 2019. 617 p.

10. Heppolette CAA, Brunnen D, Bampoe S, Odor PM. Clinical Pharmacokinetics and Pharmacodynamics of Levobupivacaine [Internet]. Clinical Pharmacokinetics. Springer International Publishing; 2020. Available from: https://doi.org/10.1007/s40262-020-008680

11. El-Shaarawy A, Asfour M, Rashwan D, Amer M, El-Menshawe S, Elkomy M. Comparison of three different concentrations of levobupivacaine for epidural labor analgesia: Clinical effect and pharmacokinetic profile. Anesth Essays Res. 2018;12(1):60.

12. Attri J, Kaur G, Kaur S, Kaur R, Mohan B, Kashyap K. Comparison of levobupivacaine and levobupivacaine with fentanyl in infraumbilical surgeries under spinal anaesthesia. Anesth Essays Res. 2015;9(2):178.

13. Behzadi M, Joukar S, Beik A. Opioids and cardiac arrhythmia: A literature review. Med Princ Pract. 2018;27(5):401-14.

14. Soma-pillay P, Nelson-piercy C, Tolppanen H, Mebazaa A. Physiological changes in pregnancy. Cardiovasc $\mathbf{J}$ Afr. 2016;27(2):89-94.

15. Willfurth I, Baik-schneditz N. Cerebral Oxygenation in Neonates Immediately after Cesarean Section and Mode of Maternal Anesthesia. 2019;

16. Sümpelmann R, Becke K, Zander R, Witt L. Perioperative fluid management in children: Can we sum it all up now. Curr Opin Anaesthesiol. 2019;32(3):384-91.

17. Davis PJ, Cladis FP. Smith's Anesthesia for Infants and Children. 9th ed. Philadelphia: Elsevier; 2017. 1131-1333 p. 18. Miao F, Feng K, Feng X, Fan L, Lang Y, Duan Q. The Analgesic Effect of Different Concentrations of Epidural Ropivacaine Alone or Combined With Sufentanil in Patients After Cesarean Section. 2021;12(February):1-9. 